\title{
Cable-Pulley Interaction with Dynamic Wrap Angle Using the Absolute Nodal Coordinate Formulation
}

\author{
Cassidy Westin, Rishad A. Irani \\ Carleton University \\ Ottawa, Canada \\ cassidy.westin@carleton.ca; rishad.irani@carleton.ca
}

\begin{abstract}
This paper presents a finite element model of a flexible cable that has been constructed to accurately model dynamic wrap angles around a static pulley. The study considers a system with a rope running over a pulley and a suspended load on one end moving in a circular arc. The model utilizes the Absolute Nodal Coordinate Formulation to define the generalized coordinates, chosen for its accurate definitions of large non-linear cable deformations. The interaction of the cable with the surface of a pulley is modeled using a contact penalty formulation. An experimental study is performed to analyze the performance of the model outputs: cable tension and wrap angle. During the testing the wrap angle had peak-to-peak oscillations of up to 122 degrees. The numerical simulation shows reasonable agreement with the experimental measurements.
\end{abstract}

Keywords: Rope Model, Finite Element Method, Absolute Nodal Coordinate Formulation, Contact Dynamics, CablePulley Interaction

\section{Introduction}

Flexible cables and pulleys are common in lifting and robotic applications and research has taken place to model the dynamics of the cables. However, few researchers examine the complex dynamic interaction between the cable and the pulley. Various discrete modeling approaches have been used to model the dynamics of flexible cables. These methods include point-mass [1], finite segment [2] and finite element methods [3]. Seo et al. [4] have noted that for cables undergoing large deformations the behavior is highly non-linear. The non-linear Absolute Nodal Coordinate Formulation (ANCF) has been shown to be accurate for modeling thin, flexible cables undergoing large deformations [5]. Both rigid body displacements and non-linear deformations of the cable can be accurately and efficiently modeled. It is thus well suited for cable contact problems, wherein the cable undergoes large deformations along the contact arc and mainly rigid body displacements elsewhere. While research extending the ANCF method to contact problems is limited, it has been applied to systems such as belt-drives [6], caternary-pantograph systems [4], as well as cable-pulley interactions [7, 8, 9].

Bulin et al. [7] use the ANCF approach to model both a statically loaded cable-pulley system and an actuated system with a mass on a slope attached to one cable end and a motor on the other. While their investigation focused mainly on the frictional aspects of the contact, the researchers also examined the normal contact force distribution as a function of the wrap angle. They demonstrate that the distribution is nonlinear and with higher cable bending stiffness the non-linearity increases. In the experimental study performed by Bulin et al., the cable experienced variations in the wrap angle of approximately 5 degrees, estimated based on the geometry provided. Takehara et al., observed similar results for a system with static, hanging loads on each end of the cable [9]. Lugris et al. analyze a similar system, comparing an analytical model and an ANCF model, but without experimental validation [8]. Experimental validation of cable models with surface contact is uncommon in the literature. The systems examined in the literature mostly consist of purely reciprocating cable motion [8,9] or systems with only small variations in the wrap angle [7]; however, there are scenarios where a dynamic cable model which can accurately depict variations in wrap angle is useful $[1,10]$. One such scenario is the cable and pulley systems for marine towed bodies where the cable is unconstrained on the submerged end and experiences highly dynamic loads and excitations. The cable undergoes both reciprocating cable motion, due to the cable being reeled in or out, and transverse motion resulting in a large varying wrap angle between the cable and pulley.

This paper presents the construction a planar ANCF model of the cable-pulley interaction utilizing various models for longitudinal and transverse stiffness and the contact forces. Unlike previous works, the presented model specifically addresses cable systems that undergo large transverse motions with a large variation in the wrap angle. Furthermore, the 
model is validated with experimental tension and position measurements. Peak-to-peak variations in the wrap angle of up to 122 degrees were tested. Section 2 of the paper provides an overview of the derivation of the equations of motion using the Absolute Nodal Coordinate Formulation. In Section 3, the computer implementation of the model and the experimental setup is discussed. The experimental and numerical results are compared in Section 4 and the paper ends with concluding remarks in Section 5.

\section{Model}

Figure 1 shows the system under consideration consisting of three main components: the cable, pulley and an external load; the load is released from rest at some initial angle $\theta$. The cable is modeled using the ANCF, where the cable is discretized into $N$ finite elements. The planar ANCF element consists of two nodes, each with four degrees of freedom. The shape of each element can then be described by a cubic polynomial with a single parameter $p$. The generalized coordinates are defined in the inertial frame and describe the absolute position and slope of the cable at each node. The interaction of the cable with pulley surface is incorporated using a contact penalty method to determine the normal contact forces. The external load is modeled as a rigid body with four generalized coordinates used to define the degrees of freedom of the load. An overview of the formulation, the derivation of the equations of motion and the incorporation of normal contact forces are described below.

\subsection{Cable Element Coordinate System}

In Figure 2, a deformed ANCF element and the corresponding undeformed element are illustrated. The absolute coordinates $\mathbf{r}$ of a point on the cable is defined using a vector of generalized coordinate $\mathbf{q}$ and a cubic shape function $\mathbf{S}(p)$ which interpolates between the nodes of each element. Thus,

$$
\mathbf{r}(p)=\mathbf{S}(p) \mathbf{q}=\left[\begin{array}{l}
x \\
y
\end{array}\right]
$$

For a fully parameterized, planar ANCF beam element of unstretched length 1, the generalized coordinates that define the degrees of freedom consist of the Cartesian coordinates $\mathrm{x}$ and $\mathrm{y}$ and the parametric slopes $\frac{\partial x}{\partial p}$ and $\frac{\partial y}{\partial p}$ at each node and are given as,

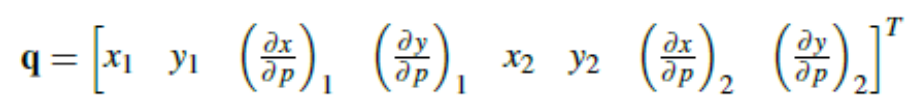

where subscripts 1 and 2 represent the respective nodes of the element or parameter values of $p=0$ and $p=1$. The shape function representing a cubic spline is

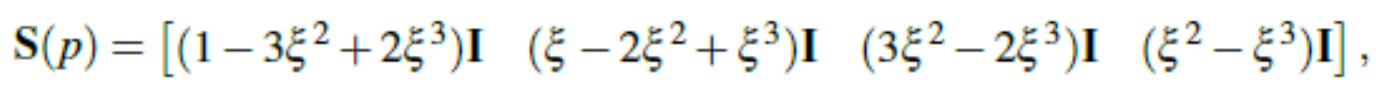

where $\mathbf{I}$ is a $2 \times 2$ identity matrix and $\xi=p / l$.

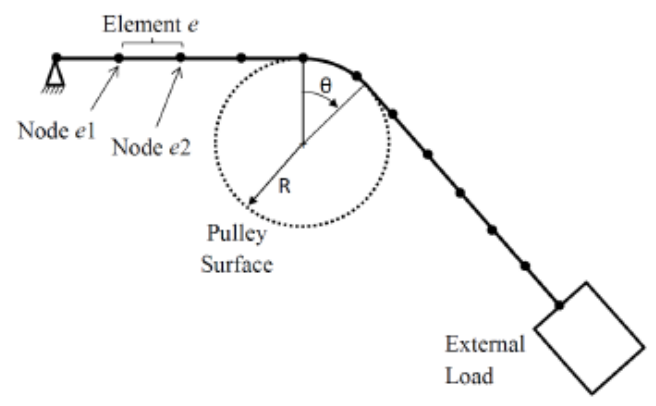

Fig. 1: Diagram of System. 


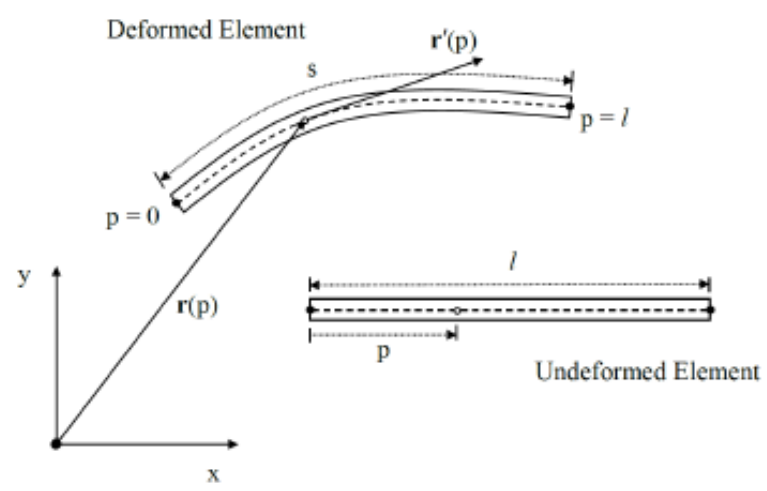

Fig. 2: Diagram of ANCF element.

\subsection{Cable Equations of Motion}

For each cable element, the standard matrix form of the Newton-Euler equations is

$$
\mathbf{M} \ddot{\mathbf{q}}+\mathbf{K q}+\mathbf{C} \dot{\mathbf{q}}-\mathbf{Q}=0,
$$

where $\mathbf{M}$ is the mass matrix, $\mathbf{K}$ is the elastic stiffness matrix, $\mathbf{C}$ is the damping matrix, $\mathbf{Q}$ is an external force vector, and $\mathbf{q}$ is the vector of generalized coordinates previously defined by Equation 2. Using the shape function $\mathbf{S}(p)$ and the generalized ANCF coordinates, the mass and stiffness matrices and external force vectors are derived as shown in following sections.

\subsubsection{Cable Mass Matrix}

In the consistent mass lumping approach used by Shabana [5], the kinetic energy of the element is defined as a function of the time derivatives of the nodal degrees of freedom defined in Equation 2. Thus,

$$
T=1 / 2 \int_{V} \rho \dot{\mathbf{r}}^{T} \dot{\mathbf{r}} d V=1 / 2 \dot{\mathbf{q}}^{T}\left[\int_{V} \rho \mathbf{S}^{T} \mathbf{S} d V\right] \dot{\mathbf{q}},
$$

where $T$ is the kinetic energy and $V$ is the volume of the cable. The mass matrix $\mathbf{M}$ can then be defined as the Hessian of the kinetic energy:

$$
\mathbf{M}=\frac{\partial^{2} T}{\partial \dot{\mathbf{q}} \partial \dot{\mathbf{q}}}=\int_{V} \rho \mathbf{S}^{T} \mathbf{S} d V
$$

\subsubsection{Cable Stiffness Matrix}

Similarly, Berzeri et al. [11] derive the stiffness matrix from the strain energy $U$ of the element given. Thus,

$$
U=\frac{1}{2} \int_{0}^{l}\left[E A \varepsilon_{l}^{2}(p)+E I \kappa^{2}(p)\right] d p,
$$

where $E$ is the Young's modulus of the cable material, A is the cross-sectional area, $I$ is the second moment of area, $\varepsilon_{1}$ is the longitudinal strain, and $k$ is the curvature of the element. The internal stiffness force is the derivative of the strain energy with respect to the coordinate vector q. The internal stiffness force vector can be separated into longitudinal and transverse components, $\mathrm{Q}_{1}$ and $\mathrm{Q}_{t}$. To simplify the computation of these forces it is assumed that the longitudinal strain $\varepsilon_{1}$ is small and also constant throughout the element. The longitudinal force as shown by Berzeri et al. [11] are 


$$
\mathbf{Q}_{\mathbf{1}}=\left[E A \varepsilon_{l} \int_{0}^{l} \mathbf{S}^{\prime T} \mathbf{S}^{\prime} d p\right] \mathbf{q}
$$

A modification is made to the longitudinal strain definition of Berzeri et al.'s model. In order to provide greater accuracy for elements with significant curvatures, as one might expect during large wrap angles, the current model defines the longitudinal strain as a function of the arc length of the element. Thus,

$$
\varepsilon_{l}(\mathbf{q})=\frac{s(\mathbf{q})-l}{l}
$$

where $s$ is the arc length and $l$ is the unstretched element length. The arc length is defined by integrating the norm of the vector $\mathbf{r}$ ' with respect to $p$ over the length of the element [12]. For the numerical solution the arc length $s$ is approximated using a numerical quadrature,

$$
s=\sum_{i=1}^{N_{s}} w_{i} \sqrt{\mathbf{r}^{\prime}\left(p_{i}\right)^{T} \mathbf{r}^{\prime}\left(p_{i}\right)} \frac{l}{N_{s}}
$$

where $i$ denotes the integration point defined along the length of the element, $N_{s}$ is the total number of integration points and $w_{i}$ is the quadrature weight. Currently, the trapezoidal rule is used with quadrature weights defined as,

$$
w_{i}= \begin{cases}0.5, & i=1, N_{s} \\ 1, & i=2,3, \ldots, N_{s}-1\end{cases}
$$

Defined by Berzeri et al. [11], the transverse stiffness force $\mathbf{Q}_{\mathrm{t}}$ is

$$
\mathbf{Q}_{t}=\left[\int_{0}^{l} E I \mathbf{S}^{\prime \prime T} \mathbf{S}^{\prime \prime} d p\right] \mathbf{q},
$$

where $I$ is the second moment of area. The general stiffness matrix $\mathbf{K}$ from Equation 4 is then given by:

$$
\mathbf{K}=\int_{0}^{l} E I \mathbf{S}^{\prime \prime T} \mathbf{S}^{\prime \prime} d p+\varepsilon_{l}(\mathbf{q}) E A \int_{0}^{l} \mathbf{S}^{T} \mathbf{S}^{\prime} d p .
$$

\subsubsection{Cable Damping Matrix}

The damping matrix $\mathrm{C}$ from Equation 4 serves both to include energy dissipation and attenuate high frequency vibrational modes. The Rayleigh-damping approach [13], wherein the damping matrix is defined as a linear combination of the mass and stiffness matrices, is used in the current model. Thus,

$$
\mathbf{C}=\alpha \mathbf{M}+\beta_{1} \mathbf{K}_{t}+\beta_{2} \mathbf{K}_{l}
$$

where $\mathbf{K}_{1}$ and $\mathbf{K}_{\mathrm{t}}$ are respectively the longitudinal and transverse components of the stiffness matrix defined in Equation 13, and $\alpha, \beta_{1}$, and $\beta_{2}$ are scalar damping coefficients.

\subsection{External Forces on Cable Element}

The vector of external forces $\mathbf{Q}$ is the summation of the gravitational body force $\mathbf{Q}_{\mathrm{g}}$, the normal contact force $\mathbf{Q}_{\mathrm{n}}$, and the kinematic constraint force $\mathbf{Q}_{\mathrm{c}}$. The derivation of the force vectors are shown in the following sections. 


\subsubsection{Constraint Forces}

The constraint forces are derived from holonomic constraint equations and define the boundary conditions and interelement connectivity. Each constraint equation is defined as a function of the generalized coordinates $\mathbf{q}$ and has the form $C(\mathbf{q})=0$. The force required to enact the constraint is

$$
\mathbf{Q}_{c}=-C_{\mathbf{q}}{ }^{T} \lambda,
$$

where the subscript q represents the Jacobian with respect to the vector of generalized coordinates and $\lambda$ is a vector of Lagrange multipliers.

The second time derivative of $C(\mathbf{q})$ is

$$
C_{\mathbf{q}} \ddot{\mathbf{q}}+\left(C_{\mathbf{q}} \dot{\mathbf{q}}\right)_{\mathbf{q}} \dot{\mathbf{q}}+2 \frac{d}{d t}\left(C_{\mathbf{q}}\right) \dot{\mathbf{q}}=0
$$

Combining Equations 4, 15 and 16, the Lagrange multipliers can be eliminated and the constraint force is expressed as

$$
\mathbf{Q}_{c}=-C_{\mathbf{q}}{ }^{T}\left[C_{\mathbf{q}} \mathbf{M}^{-1} C_{\mathbf{q}}{ }^{T}\right]^{+}\left[C_{\mathbf{q}} \mathbf{a}-\left(C_{\mathbf{q}} \dot{\mathbf{q}}\right)_{\mathbf{q}} \dot{\mathbf{q}}-2 \frac{d}{d t}\left(C_{\mathbf{q}}\right) \dot{\mathbf{q}}\right],
$$

where + represents the Moore-Penrose pseudo-inverse and a is the associated accelerations of the unconstrained system,

$$
\mathbf{a}=\mathbf{M}^{-1}\left(\mathbf{Q}_{g}+\mathbf{Q}_{n}-\mathbf{C} \dot{\mathbf{q}}-\mathbf{K q}\right)
$$

Used within the construction of $C_{\mathrm{q}}$, the connectivity between two elements, $i$ and $j$, is

$$
C_{i, j}=\mathbf{r}_{i}(l)-\mathbf{r}_{j}(0)=\mathbf{S}(l) \mathbf{q}_{i}-\mathbf{S}(0) \mathbf{q}_{j}=0
$$

\subsubsection{Gravitational Force}

The gravitational force on each element is

$$
\mathbf{Q}_{g}=\rho \int_{V} \mathbf{S}^{T} \mathbf{g} d V
$$

where $\rho$ is the cable density, $V$ is the elemental cable volume and $g=[0-9.81]^{\mathrm{T}} \mathrm{m} / \mathrm{s}^{2}$ is the vector of the gravitational acceleration in absolute coordinates.

\subsubsection{Cable-Pulley Contact Forces}

To model the cable-pulley interaction, a contact penalty is used. The normal force vector $\mathbf{F}_{n}$ acting at a single point on the element is defined using the Hunt-Crossley model [8], which represents the surface as a non-linear spring-damper

$$
\mathbf{F}_{n}=K_{n} \delta^{b}(1+D \dot{\delta}) \hat{\mathbf{r}}
$$

where $\hat{\mathbf{r}}$ is the unit vector normal to the pulley surface at the point of contact, $K_{n}$ is the contact stiffness, $\delta$ is the relative "penetration" of the node into the surface, $D$ is a damping coefficient and $b$ is a positive constant with a value between 1 and 1.5 from the Hertz contact theory [14]. Selecting the origin at the center of the pulley with radius $R$ and noting that a negative value of the penetration has no physical meaning, the penetration and penetration velocity are defined [7]: 


$$
\begin{gathered}
\delta= \begin{cases}\sqrt{\mathbf{r}^{T} \mathbf{r}}-R & \text { if } \sqrt{\mathbf{r}^{T} \mathbf{r}}<R \\
0 & \text { otherwise }\end{cases} \\
\dot{\delta}=-\dot{\mathbf{r}}^{T} \hat{\mathbf{r}}
\end{gathered}
$$

The contact force is distributed along the length of the element, thus the total contact force $\mathbf{Q}_{n}$ in the element coordinates is approximated by a numerical quadrature,

$$
\mathbf{Q}_{n}=\sum_{i=1}^{N_{k}} w_{i} \mathbf{F}_{n}\left(p_{i}\right)^{T} \mathbf{S}\left(p_{i}\right),
$$

where $i$ denotes the integration point, $N_{k}$ is the total number of integration points and wi is the quadrature weight. As with the arc length in Section 2.2.2, the trapezoidal rule is used with the quadrature weights defined in Equation 11.

\subsection{External Load and Multibody System}

For the system under consideration, Figure 1, one end of the rope is attached to an external load, modeled as a rigid body with mass $m_{L}$. The vector of generalized coordinates consisting of the absolute coordinates $x_{P}$ and $y_{P}$ of the attachment point and the absolute coordinates $x_{I}$ and $y_{I}$ of the load's center of inertia is

$$
\mathbf{q}_{L}=\left[\begin{array}{llll}
x_{P} & y_{P} & x_{I} & y_{I}
\end{array}\right]^{T}
$$

The mass matrix of the rigid body, derived by Jalon and Bayo [15], is implemented here as,

$$
\mathbf{M}_{L}=\left[\begin{array}{cc}
2 m_{L}\left(1-\frac{L_{P, G}}{L_{P}}\right) \mathbf{I} & m_{L}\left(\frac{L_{P, G}}{L_{P, I}}-1\right) \mathbf{I} \\
m_{L}\left(\frac{L_{P, G}}{L_{P, I}}-1\right) \mathbf{I} & m_{L} \mathbf{I}
\end{array}\right],
$$

where $L_{P, G}$ and $L_{P, I}$ are the distances between the cable attachment point and the centers of gravity and inertia, respectively, and $I$ is a $2 \times 2$ identity matrix. Points $P$ and $I$ are constrained using the holonomic constraint equation

$$
C_{P, I}=\sqrt{r_{P, I}^{T} r_{P, I}}-L_{P, I}=0
$$

The constraint forces for the rigid body are calculated in the same manner described in Section 2.3.1, using $C_{P, I}$ in place of $C$.

The multibody system under consideration is made up of $N$ cable elements and the rigid body at the end of the cable. The subscript $e=1,2, \ldots, N$ is used to denote individual elements. To form the coordinate vector $\overline{\boldsymbol{q}}$ for the entire system the coordinate vectors for the cable elements $\mathbf{q}_{\mathrm{e}}$ and the rigid body $\mathbf{q}_{\mathrm{L}}$ are concatenated vertically; thus,

$$
\overline{\mathbf{q}}=\left[\begin{array}{lllll}
\mathbf{q}_{1}{ }^{T} & \mathbf{q}_{2}{ }^{T} & \ldots & \mathbf{q}_{N}^{T} & \mathbf{q}_{L}^{T}
\end{array}\right]^{T} .
$$

The resulting system equations of motion can then be written as,

$$
\overline{\mathbf{M}} \frac{d^{2} \overline{\mathbf{q}}}{d t^{2}}+\overline{\mathbf{C}} \frac{d \overline{\mathbf{q}}}{d t}+\overline{\mathbf{K}} \overline{\mathbf{q}}-\overline{\mathbf{Q}}=0
$$


where $\overline{\boldsymbol{M}}, \overline{\boldsymbol{C}}$, and $\overline{\boldsymbol{K}}$ are block diagonal system mass, damping and stiffness matrices and $\overline{\boldsymbol{Q}}$ contains the summations of the external forces on each element concatenated vertically.

In the following section the implementation of the model and the setup of the experimental validation are described.

\section{Model Implementation and Validation \\ 3.1. Computer Implementation}

The implementation of the model, performed in MATLAB, consists of two stages, a pre-processing stage and numerical integration of the equations of motion. In the pre-processing stage, the shape function is integrated symbolically to determine the system mass matrix $\bar{M}$, gravitational force vector $\bar{Q} \mathrm{~g}$, and constant components of the system stiffness matrix $\bar{K}$. The shape function and its derivative are evaluated at a set of predefined points, which are required to calculate the element strains and contact penetrations. In the second stage of the solution, MATLAB's first order ODE solve, ode15s, is used to perform the numerical integration. The physical parameters of the model which were investigated in this study are found in Table 1

Table 1: Model parameters.

\begin{tabular}{|c|c|c|c|}
\hline Configuration & 1 & 2 & 3 \\
\hline Pulley radius, $R(\mathrm{~cm})$ & 4.76 & 4.76 & 4.76 \\
Rope diameter, $d(\mathrm{~cm})$ & 0.953 & 0.953 & 0.953 \\
Rope density, $\rho(\mathrm{g} / \mathrm{cm})$ & 0.59 & 0.59 & 0.59 \\
Rope modulus of elasticity, $E(\mathrm{GPa})$ & 3.0 & 3.0 & 3.0 \\
Rope second moment of area, $I\left(\mathrm{~m}^{4}\right)$ & $4.0 \times 10^{-14}$ & $4.0 \times 10^{-14}$ & $4.0 \times 10^{-14}$ \\
External load mass, $m_{L}(\mathrm{~kg})$ & 9.43 & 18.50 & 27.57 \\
External load length, $L_{P, I}(\mathrm{~cm})$ & 26.9 & 25.9 & 24.5 \\
External load length, $L_{P, G}(\mathrm{~cm})$ & 25.8 & 24.5 & 23.2 \\
Initial wrap angle, $\theta_{0}(\mathrm{deg})$ & 34.9 & 40.4 & 49.4 \\
\hline
\end{tabular}

Due to the complexity of the contact penalty forces of the model and the sensitivity to the initial conditions, it is difficult to determine exact initial conditions of each node analytically. Thus, a preliminary estimate of the initial nodal configuration is assumed while a forcing function is applied to the external load such that the system is driven towards the initial nominal wrap angle and the nodal velocities converge to zero. Once the condition for the nodal velocities and wrap angle are met, a stable simulation can commence.

A convergence study was performed using the parameters listed for Configuration 1 in Table 1 . Simulations were performed to determine the cable tension and wrap angle while varying the number of elements from 10 to 25 elements. Each simulation was performed with 10 integration points per element. Figure 3 a shows the angle of the cable from the horizontal as a function of time for 10 and 20 element simulations. The 10 element simulation is represented by the red line, while the 20 element simulation is represented by the blue line. It was found that the rope motion, specifically the angle of the rope from the horizontal axis, has a low dependence on the number of elements. Figure $3 b$ shows the simulated cable tension for the same two configurations as a function of time. The 10 element simulation is represented by the red line, while the 20 element simulation is represented by the blue line. Spurious low frequency oscillations in the tension are observed when 10 are used. The amplitude of the oscillations is reduced as the number of elements is increased to 15 , and becomes negligible with 20 elements. With 25 elements, there is no further reduction in the amplitude of the vibrations, but a 59\% increase in the computation time. Thus, 20 elements were used for subsequent simulations. Figure $3 \mathrm{a}$ also indicates that the model implementation is able to capture a large dynamic variation of the wrap angle (122 degrees peak-to-peak) something which previous studies have not explicitly shown [7]. 


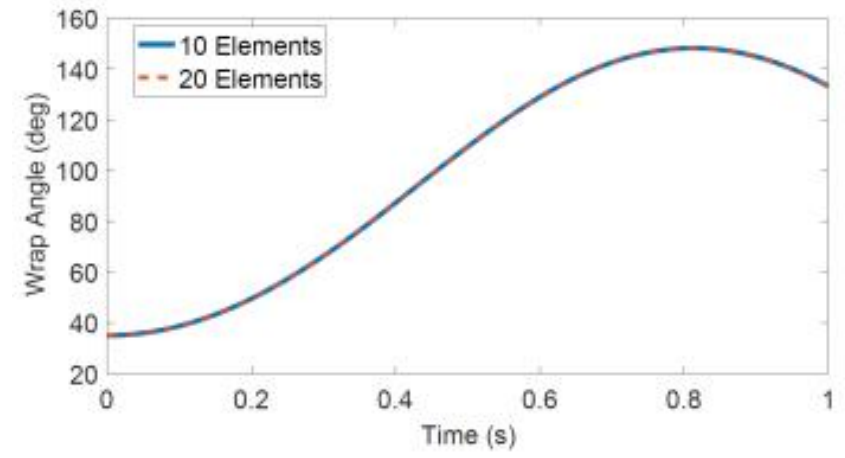

(a)

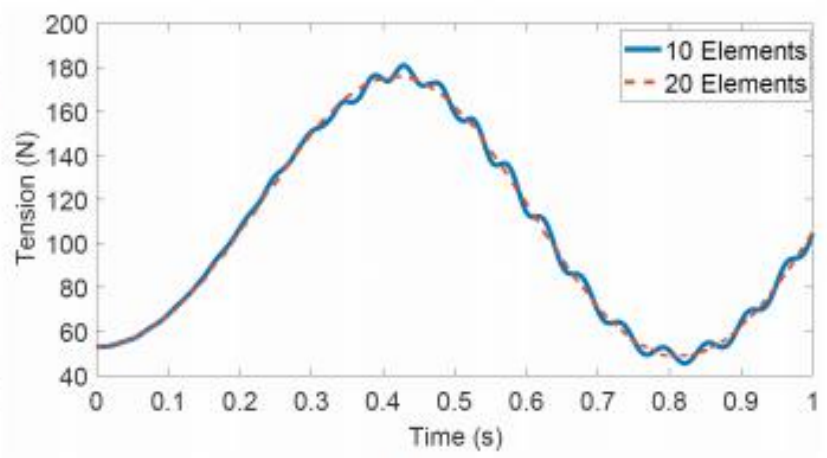

(b)

Fig. 3: Simulated wrap angle and tension as a function of time using 10 and 20 elements.

\subsection{Experimental Setup and Parameters}

In order to validate the model an experiment was performed. Consistent with Figure 1, the experiment used a small pulley and a nylon rope fixed at one end and attached to a load at the other. The load was released from an initial angle, measured from the horizontal, with the rope taut. The tension was measured using a $100 \mathrm{~kg}$ load cell. The angle of the rope relative to the horizontal axis was measured via a vision system to determine the wrap angle of the cable on the pulley. Colored markers were placed at two points along the rope and camera footage of the rope motion was collected at 60 frames per second.

The recorded frames were converted to Hue-Saturation-Value (HSV) colormaps, then each pixel with a hue and saturation value within predefined ranges was identified. The coordinates of each matching pixel were then averaged to find the centroid of each marker. This analysis was performed using the image processing tools in MATLAB. A low-pass Butterworth filter was applied to both the tension and angle measurements. The experiment was repeated for varying loads and initial wrap angles. The parameters of the system recorded during the experiments are given in Table 1. These values were also used to perform the matching simulations. The modulus of elasticity of Nylon composites ranges from 2 to $4 \mathrm{GPa}$ [16], thus a representative value of $3 \mathrm{GPa}$ was used. Due to the complex bending behavior of fibrous rope, the second moment of area is significantly smaller than that of a solid. Similar to the convergence shown by Bulin et al. [7], the second moment of area was selected by lowering the value until the contact forces converged to a constant distribution and was held constant at $4.0 \times 10^{-14} \mathrm{~m}^{4}$ for all simulations. The rope length is measured along the centerline from the top of the pulley to the load attachment point and was $42 \mathrm{~cm}$. A comparison of the experimental measurements and the simulated results is provided in the following sections.

\subsection{Simulation and Experiment Comparison}

A comparative analysis was performed for three test cases using the parameters listed in Table 1 and the representative results are displayed in Figure 4. Ten seconds of simulated motion was recorded for each run and 20 elements were used. Each element was further discretized to 10 equal increments of the parameter p for evaluating the element strain and contact distribution. The damping parameters of Equations 14 and 21 were increased until the high frequency vibration modes were attenuated and stability of the numerical solution was obtained. The final values used in the simulations were: $\alpha=0 \mathrm{~s}^{-1}, \beta_{1}=1 \times 10^{-2} \mathrm{~s}, \beta_{2}=1 \times 10^{-4}$, and $\mathrm{D}=1 \mathrm{~s} / \mathrm{m}$ 


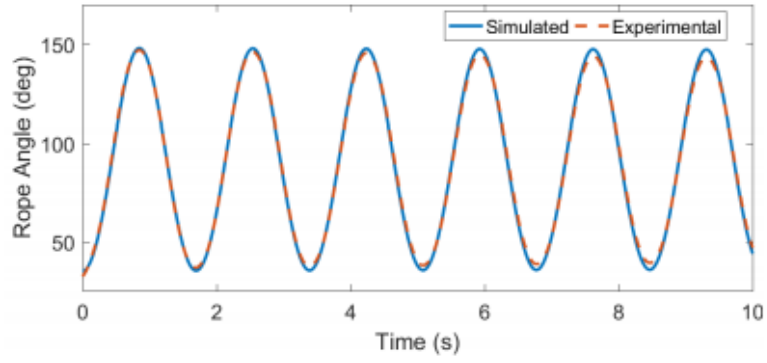

(a)

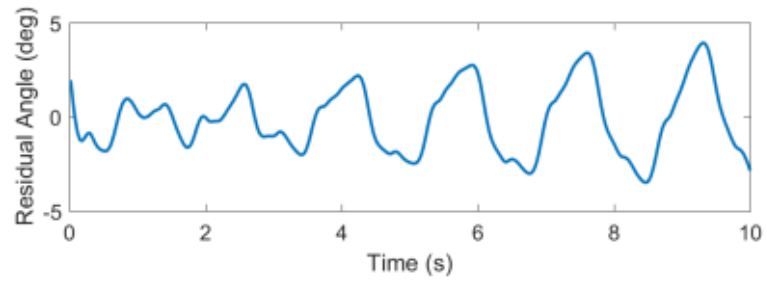

(b)

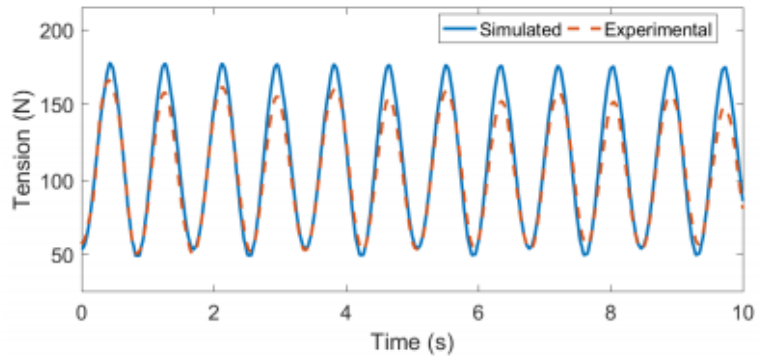

(c)

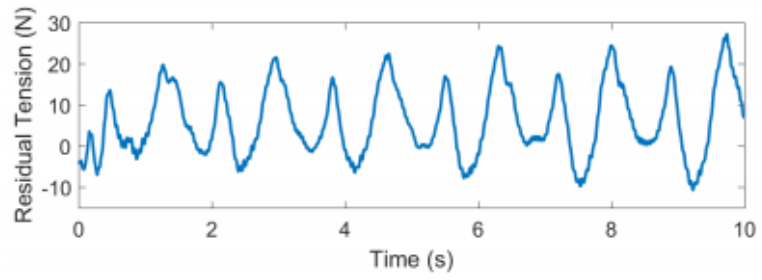

(d)

Fig. 4: Configuration 1: (a) Experimental and simulated rope angle measured from horizontal, (b) residual error of simulated rope angle compared to experimental measurement, (c) experimental and simulated rope tension, and (d) residual error of simulated rope tension compared to experimental measurement.

Figure 4a plots the numerical, a solid blue line, and experimental results, dashed orange line, of the wrap angle in degrees as a function of time for Configuration 1. Figure $4 \mathrm{~b}$ plots the error between the simulated wrap angle and the experimental measurements over the same time frame. The standard deviation of the error was determined to be 1.76 degrees 7 with a maximum error of 3.90 degrees as the wrap angle varied between 34.9 and 147 degrees. Similarly, the standard deviation of the error was 2.71 degrees with a maximum error of 5.27 degrees as the wrap angle varied between 40.4 and 139 degrees for Configuration 2. The standard deviation of the error was 2.97 degrees with a maximum error of 5.86 degrees as the wrap angle varied between 49.4 and 130 degrees for Configuration 3. These results indicate a good agreement of the model implementation to the physical results over the given time period.

Using the same line types, Figure $4 \mathrm{c}$ compares the numerical and experimental cable tension while Figure $4 \mathrm{~d}$ shows the difference between the simulated and experimental tension as a function of time for Configuration 1 . The standard deviation of the residuals was determined to be $8.58 \mathrm{~N}$ with a maximum error of $27.2 \mathrm{~N}$. The measured tension varied between 49 and $167 \mathrm{~N}$. Similarly, the standard deviation of the tension error was $20.3 \mathrm{~N}$ with a maximum error of $54.9 \mathrm{~N}$ for Configuration 2 where the measured tension varied between $121 \mathrm{~N}$ and $297 \mathrm{~N}$. The standard deviation of the tension error was $25.3 \mathrm{~N}$ with a maximum error of $64.2 \mathrm{~N}$ for Configuration 3 where the measured tension varied between 204 and $370 \mathrm{~N}$.

\section{Conclusion}

In this paper, a finite element model based on the Absolute Nodal Coordinate Formulation was proposed to simulate the motion and elastic behavior of a cable and pulley system. The model was developed specifically for cases where there are large dynamic variations in the wrap angle and cable tension. A contact penalty method was used to model the interaction of the cable with the surface of a pulley. An experiment with a rope, pulley and a suspended load was performed. After releasing the load from an initial position and allowing it to swing, the cable orientation and tension were measured. During the testing the wrap angle had peak-to-peak swings of up to 122 degrees. The standard deviations of the rope angle error between the simulation and experimental were 1.76, 2.71 and 2.97 degrees for the three configurations tested. The standard deviations of the rope tension error were 8.58, 20.3 and $25.3 \mathrm{~N}$. Future work will focus on reducing the error in the tension and extending the model to a marine towing system with reciprocating cable motion and motion of the pulley reference frame. 


\section{References}

[1] C. Calnan, R. J. Bauer, and R. A. Irani, "Controller design and motion compensation for marine towed bodies," in OCEANS 2016 MTS/IEEE Monterey, 2016.

[2] J. W. Kamman and R. L. Huston, "Modeling of variable length towed and tethered cable systems," J. Guidance, Control and Dynamics, vol. 22, no. 4.

[3] J. Ketchman and Y. Lou, "Application of the finite element method to towed cable dynamics," in OCEAN 75 Conf., pp. 98-107.

[4] J.-H. Seo et al., "Dynamic analysis of a pantograph-catenary system using absolute nodal coordinates," Veh. Syst. Dyn., vol. 44, no. 8, pp. 615-630, Aug. 2006.

[5] A. A. Shabana, "Flexible multibody dynamics: Review of past and recent developments," Multibody System Dynamics, vol. 1, no. 2, pp. 189-222, 1997.

[6] K. S. Kerkkanen, D. Garca-Vallejo, and A. M. Mikkola, "Modeling of belt-drives using a large deformation finite element formulation," Nonlinear Dyn., vol. 43, no. 3, pp. 239-256, Feb. 2006.

[7] R. Bulın et al., "Nonlinear dynamics of a cablepulley system using the absolute nodal coordinate formulation," Mechanics Research Commun., 2017.

[8] Lugris, Urbano et al. "Efficient And accurate simulation of the cable-pulley interaction in weight-lifting machines". $I^{\text {st }}$ Joint Int. Conference On Multibody System Dynamics. Lappeenranta, Finland. 189-190, 2017.

[9] S. Takehara, M. Kawarada, and K. Hase, "Dynamic contact between a wire rope and a pulley using Absolute Nodal Coordinate Formulation," Machines, vol. 4, no. 1, p. 4, 2016.

[10] C. Calnan, "Set-point algorithms for active heave compensation of towed bodies," M.S. Thesis, Dept. Mech. Eng., Dalhousie Univ., Halifax, Canada, 2016.

[11] M. Berzeri and A. A. Shaban, "Development of simple models for the elastic forces in the Absolute Nodal Co ordinate Formulation," Journal of Sound and Vibration, vol. 235, no. 4, pp. 539565, Aug. 2000.

[12] M. Ciftci, "Flexible multibody analysis using Absolute Nodal Coordinate Formulation," M.S. Thesis, Middle East Technical Univ., Ankara, Turkey, 2014.

[13] L. Kubler, P. Eberhard, and J. Geisler, "Flexible multibody systems with large deformations and nonlinear structural damping using Absolute Nodal Coordinates," Nonlinear Dyn., vol. 34, no. 1/2, pp. 31-52, Oct. 2003.

[14] P. Flores, et al., "Influence of the contact-impact force model on the dynamic response of multibody systems, " in Proc. Institution of Mechanical Engineers, Part K, J. of Multibody Dynamics, vol. 220, pp. 21-34, Jan. 2006.

[15] J. G. D. Jalon and E. Bayo, Kinematic and dynamic simulation of multibody systems: the realtime challenge. New York: Springer-Verlag, 1994.

[16] H. Unal et al., "Mechanical behavior of nylon composites containing talc and kaolin," J. of Appl. Polymer Science, vol. 88, no. 7, pp. 1694-1697, Apr. 2003. 